\title{
Rainbow-free 3-colorings in abelian groups
}

\author{
Amanda Montejano ${ }^{1,2}$ \\ Dept. Matemàtica Aplicada 4 \\ Univ. Politècnica de Catalunya \\ Barcelona, Spain \\ Oriol Serra ${ }^{3}$ \\ Dept. Matemàtica Aplicada 4 \\ Univ. Politècnica de Catalunya \\ Barcelona, Spain
}

\begin{abstract}
A 3-coloring of an abelian group $G$ is rainbow-free if there is no 3-term arithmetic progression with its members having pairwise distinct colors. We describe the structure of rainbow-free colorings of abelian groups. This structural description proves a conjecture of Jungić et al. on the size of the smallest chromatic class of a rainbow-free coloring of cyclic groups.

Keywords: Anti-Ramsey Theory, Arithmetic Progressions.

\footnotetext{
1 Supported by the Ministry of Science and Innovation of Spain under project MTM200806620-C03-01 and by the CONACYT agency from the government of Mexico.

2 Email: amanda@ma4.upc.edu

3 Email: oserra@ma4.upc.edu
} 


\section{Introduction}

Let $c$ be a coloring of a set $X$. A subset $Y \subset X$ is rainbow under $c$ if the coloring assigns pairwise distinct colors to the elements of $Y$. The study of the existence of rainbow structures falls into the anti-Ramsey theory initiated by Erdös, Simonovits and Sós [1]. Arithmetic versions of this theory where initiated by Jungić, Licht, Mahdian, Nešetřil and Radoičić [2] where the authors study the existence of rainbow 3-term arithmetic progressions in colorings of cyclic groups and integer intervals.

A 3-term arithmetic progression $A P(3)$ in an abelian group is a triple $(x, y, z)$ with $x+y=2 z$. In this setting we say that a coloring is rainbowfree if there are no rainbow $A P(3)$ 's. Among other results the authors in [2] proved that if the smaller class in a 3 -coloring of the cyclic group $\mathbb{Z} / n \mathbb{Z}$ has size greater than $n / 6$, then there exists a rainbow $A P(3)$. For $n$ divisible by 6 this condition is tight, but for other values of $n$ it is possible to obtain weaker assumptions. Let $m(n)$ be the largest integer $m$ for which there is a rainbowfree 3 -coloring of $\mathbb{Z} / n \mathbb{Z}$ such that the cardinality of the smaller color class is $m$. Jungić, Nešetřil and Radoičić [3] formulated the following conjecture. Let $\mathcal{P}_{0}$ be the set of primes for which 2 has either multiplicative order $p-1$, or multiplicative order $(p-1) / 2$ with $(p-1) / 2$ odd. Let $\mathcal{P}_{1}$ be the set of remaining primes.

Conjecture 1.1 ([3]) Let $n$ be an integer which is not a power of 2. Let $p$ denote the smallest odd prime factor of $n$ in $\mathcal{P}_{0}$ and let $q$ be the smallest odd prime factor of $n$ in $\mathcal{P}_{1}$. Then

$$
m(n)=\left\lfloor\frac{n}{\min \{2 p, q\}}\right\rfloor .
$$

For $n=2^{m}$ and primes in $\mathcal{P}_{0}$ it had been already proved in [2] that $m(n)=0$. Actually these two cases characterize the possible values of $n$ for which every 3 -coloring has a rainbow $A P(3)$.

Theorem 1.2 (Jungić et al. [2] (2003)) For every integer $n$, there is a rainbow-free 3 -coloring of $\mathbb{Z} / n \mathbb{Z}$ with non-empty color classes, if and only if $n$ does not satisfy any of the following conditions:

(a) $n$ is a power of 2.

(b) $n \in \mathcal{P}_{0}$.

For general $n$ the authors of [2] proved the following bounds. 
Theorem 1.3 (Jungić et al. [2] (2003)) Let $n$ be not a power of 2, $q$ be the smallest prime factor of $n$, and $r$ be the smallest odd prime factor of $n$, then:

$$
\left\lfloor\frac{n}{2 r}\right\rfloor \leq m(n) \leq \min \left(\frac{n}{6}, \frac{n}{q}\right)
$$

Thus concerning cyclic groups of prime order, it follows from Theorem 1.3 that $m(p) \leq 1$ and, from Theorem 1.2, that if $p \in \mathcal{P}_{0}$ then there are no rainbow-free 3 -coloring of $\mathbb{Z} / p \mathbb{Z}$ with non-empty color classes.

\section{Main results}

In this paper we give a structural result of rainbow-free 3-colorings on general abelian groups of odd order. The following Proposition describes the structure of a rainbow-free 3-coloring, of an abelian group of odd order, when there is a color class with just one element.

Proposition 2.1 Let $G$ be a finite abelian group of odd order $n$, and let $c$ be a 3-coloring of $G$ with color classes $A, B, C$ such that $|A|=1$. Then $c$ is rainbow-free if and only if, up to translation, every $X \in\{A, B, C\}$ satisfies $X=2 X=-X$.

Our main result Theorem 2.2 below states that a rainbow-free 3-coloring of an abelian group $G$ of odd order is essentially obtained by lifting a rainbowfree 3-coloring with a color class of size one from a quotient group.

Theorem 2.2 Let $G$ be a finite abelian group of odd order $n$ and let $c$ be a 3 -coloring of $G$ with non-empty color classes $A, B, C$. Then c is rainbow-free if and only if, up to translation, there is a proper subgroup $H<G$ and a color class, say $A$, such that:

(i) $A \subseteq H$, and the 3 -coloring induced in $H$ is rainbow-free,

(ii) $\widetilde{B}+H=\widetilde{B}$ and $\widetilde{C}+H=\widetilde{C}$,

(iii) $\widetilde{B}=-\widetilde{B}=2 \widetilde{B}$ and $\widetilde{C}=-\widetilde{C}=2 \widetilde{C}$.

where $\widetilde{B}=B \backslash H$ and $\widetilde{C}=C \backslash H$.

The proof of the "if" part of the Theorem can be easily checked by considering the 3 -coloring of $G / H$ with chromatic classes $(A / H, \widetilde{B} / H, \widetilde{C} / H)$ and Proposition 2.1. For the "only if" part we use some results in Additive Combinatorics on the structure of sets with small sumset. Let $c$ be a 3 -coloring of an abelian group $G$ of odd order with color classes $A, B, C$. If 
$|A+B| \geq|A|+|B|+1$ then (since $n=|G|$ is odd) we have $|A+B|>|G|-|2 \cdot C|$, where $A+B=\{a+b, a \in A, b \in B\}$ denotes the Minkowski sum of $A$ and $B$, and $2 \cdot C=\{2 c, c \in C\}$. Therefore the coloring has a rainbow $A P(3)$. It follows from Kneser's theorem (see e.g. [6]) that the case $|A+B| \leq|A|+|B|-1$ can be reduced to the case of equality. The Kemperman Structure Theorem [4] provides precise information on the structure of sets $A, B$ in an abelian group verifying $|A+B|=|A|+|B|-1$. This deep structural result has been recently extended by Grynkiewicz [5] to handle the case where $|A+B|=|A|+|B|$. These two structural results are used here to prove Theorem 2.2.

The description of rainbow-free 3-colorings of abelian groups of odd order can be used to prove Conjecture 1.1 for cyclic groups. Actually the Conjecture holds for general abelian groups of odd order.

Corollary 2.3 Let $G$ be an abelian group of odd order $n$. Let $p$ denote the smallest prime factor of $n$ in $\mathcal{P}_{0}$ and let $q$ be the smallest prime factor of $n$ in $\mathcal{P}_{1}$. If $\{A, B, C\}$ is a rainbow-free 3 -coloring of $G$ then

$$
\min \{|A|,|B|,|C|\} \leq\left\lfloor\frac{n}{\min \{2 p, q\}}\right\rfloor .
$$

Moreover, there are rainbow-free 3-colorings of $G$ for which equality holds.

Proof. We first prove (1). By Theorem 2.2 (i), one color class, say $A$, is contained in a subgroup $H<G$ and

$$
|A| \leq|H| \leq \max \left\{\frac{n}{p}, \frac{n}{q}\right\} .
$$

Thus we may assume that $|H|=\frac{n}{p}$ and $G / H$ is a cyclic group of prime order $p \in \mathcal{P}_{0}$, otherwise we are done. By Theorem 2.2 (ii), each of the two sets $\widetilde{B}=B \backslash H$ and $\widetilde{C}=C \backslash H$ is a (possibly empty) union of $H$-cosets.

Suppose that both sets $\widetilde{B}$ and $\widetilde{C}$ are nonempty. Since $p \in \mathcal{P}_{0}$ it follows from Theorem 1.2 that the 3 -coloring of $G / H \simeq \mathbb{Z} / p \mathbb{Z}$ with color classes $A^{\prime}=\{0\}$, $B^{\prime}=\widetilde{B} / H$ and $C^{\prime}=\widetilde{C} / H$ contains a rainbow $A P(3)$ which must be of the form $\{-x, 0, x\}$ for some $x \in G / H$. But this contradicts that $\widetilde{B}=-\widetilde{B}$ and $\widetilde{C}=-\widetilde{C}$, Theorem 2.2 (iii). Hence $G \backslash H$ is monochromatic and thus $H$ contains two colors. It follows that $\min \{|A|,|B|,|C|\} \leq\left\lfloor\frac{n}{2 p}\right\rfloor$.

Let us show that there are rainbow-free 3 -colorings of $G$ for which equality holds in (1).

If $2 p \leq q$ then choose a subgroup $H<G$ with cardinality $\frac{n}{p}$, consider a partition $A \cup B=H$ where $|A|=\left\lfloor\frac{n}{2 p}\right\rfloor$, and let $C=G \backslash H$. If $q<2 p$ then 
choose a subgroup $H<G$ with cardinality $\frac{n}{q}$ and let $B^{\prime}=\left\{2,2^{2}, \ldots, 2^{b}=1\right\}$ in the cyclic group $G / H \simeq \mathbb{Z} / q \mathbb{Z}$. Since $q \notin \mathcal{P}_{0}$, the set $C^{\prime}=G / H \backslash\left(B^{\prime} \cup\right.$ $\left.-B^{\prime} \cup\{0\}\right)$ is nonempty. Define the coloring $A=H, B=\pi^{-1}\left(B^{\prime} \cup-B^{\prime}\right)$ and $C=\pi^{-1}\left(C^{\prime} \cup-C^{\prime}\right)$, where $\pi: G \rightarrow G / H$ denotes the natural projection. In both cases the coloring satisfies parts (i), (ii) and (iii) of Theorem 2.2 and therefore it is rainbow-free.

Once proved for abelian groups of odd order one can show that Conjecture 1.1 holds for cyclic groups as well by induction on the largest power of 2 dividing the order of the group. However the Conjecture does not hold for general abelian groups of even order. We obtain a counterexample by means of the Klein group $\mathbb{Z}_{2} \times \mathbb{Z}_{2}$. Let $G:=H \times\left(\mathbb{Z}_{2} \times \mathbb{Z}_{2}\right)$ where $|H|$ is not a power of 2. Consider the following 3-coloring of $G$ : let the subgroup $H$ be colored by $A$, color one of the three remaining $H$-cosets of $G$ by $B$ and the remaining two by $C$. This coloring is rainbow-free, since a 3 -term arithmetic progression in $\mathbb{Z}_{2} \times \mathbb{Z}_{2}=G / H$ is of the form $(x, x, y)$. however the smaller color class has cardinality $|H|=\frac{n}{4}>\min \left\{\frac{n}{2 p}, \frac{n}{q}\right\}$ where $|G|=n, p$ is the smallest odd prime factor of $n$ in $\mathcal{P}_{0}$, and $q$ is the smallest odd prime factor of $n$ in $\mathcal{P}_{1}$.

\section{References}

[1] P. Erdős, M. Simonovits, V. Sós, Anti-Ramsey theorems. Infinite and finite sets, Colloq. Math. Soc. Janos Bolyai, Vol. 10, 633-643, 1975

[2] V. Jungić, J. Licht, M. Mahdian, J. Nešetřil and R. Radoičić. Rainbow arithmetic progressions and anti-Ramsey results. Combin. Probab. Comput. 12 (2003), no. $5-6,599-620$.

[3] V. Jungić, J. Nešetřil and R. Radoičić. Rainbow Ramsey Theory. Integers: Electronic Journal of Combinatorial Number Theory 5(2) A9. (2005).

[4] J.H.B. Kemperman. On small sumsets in an abelian group. Acta Math 103 (1960) 63-88.

[5] D.J. Grynkiewicz. A step beyond Kemperman's Structure Theorem, Mathematika (to appear 2009).

[6] T. Tao, V. Vu. Additive combinatorics. Cambridge Studies in Advanced Mathematics, 105. Cambridge University Press, Cambridge, 2006. 